\title{
Cooperating, congenital neutropenia-associated Csf3r and Runx1 mutations activate pro-inflammatory signaling and inhibit myeloid differentiation of mouse HSPCs
}

\author{
Malte Ritter ${ }^{1}$ - Maksim Klimiankou ${ }^{1}$ - Olga Klimenkova ${ }^{1}$ - Axel Schambach ${ }^{2,3}$ - Dirk Hoffmann ${ }^{2}$ - Amy Schmidt ${ }^{4}$. \\ Lothar Kanz ${ }^{1}$ - Daniel C. Link ${ }^{4} \cdot$ Karl Welte $^{5}$ - Julia Skokowa ${ }^{1}$ (1)
}

Received: 3 March 2020 / Accepted: 20 July 2020 / Published online: 3 August 2020

(C) The Author(s) 2020, corrected publication 2021

\begin{abstract}
Patients with the pre-leukemia bone marrow failure syndrome called severe congenital neutropenia (CN) have an approximately $15 \%$ risk of developing acute myeloid leukemia (AML; called here CN/AML). Most CN/AML patients co-acquire $C S F 3 R$ and RUNX1 mutations, which play cooperative roles in the development of AML. To establish an in vitro model of leukemogenesis, we utilized bone marrow lin ${ }^{-}$cells from transgenic C57BL/6-d715 $C s f 3 r$ mice expressing a CN patient-mimicking truncated CSF3R mutation. We transduced these cells with vectors encoding RUNX1 wild type (WT) or RUNX1 mutant proteins carrying the R139G or R174L mutations. Cells transduced with these RUNXI mutants showed diminished in vitro myeloid differentiation and elevated replating capacity, compared with those expressing WT RUNX1. mRNA expression analysis showed that cells transduced with the RUNX1 mutants exhibited hyperactivation of inflammatory signaling and innate immunity pathways, including IL-6, TLR, NF-kappaB, IFN, and TREM1 signaling. These data suggest that the expression of mutated RUNX1 in a $C S F 3 R$-mutated background may activate the pro-inflammatory cell state and inhibit myeloid differentiation.
\end{abstract}

Keywords Severe congenital neutropenia $\cdot$ Pre-leukemia bone marrow failure syndrome $\cdot$ G-CSFR mutations $\cdot$ RUNX1 mutations

Electronic supplementary material The online version of this article (https://doi.org/10.1007/s00277-020-04194-0) contains supplementary material, which is available to authorized users.

Julia Skokowa

Julia.Skokowa@med.uni-tuebingen.de

1 Division of Translational Oncology, Department of Hematology, Oncology, Clinical Immunology and Rheumatology, University Hospital Tübingen, Otfried-Müller-Straße 10, 72076 Tübingen, Germany

2 Institute of Experimental Hematology, Hannover Medical School, Hannover, Germany

3 Division of Hematology/Oncology, Boston Children's Hospital, Harvard Medical School, Boston, MA, USA

4 Division of Oncology, Section of Stem Cell Biology, Washington University Medical School, St. Louis, MO, USA

5 The University Children's Hospital Tübingen, Tübingen, Germany

\section{Introduction}

Patients with the inborn pre-leukemia bone marrow failure syndrome called severe congenital neutropenia $(\mathrm{CN})$ have a very low level (less than 500 cells $/ \mu$ l blood) or even a complete lack of mature neutrophilic granulocytes in the peripheral blood which is caused by blockade of the terminal differentiation of bone marrow myeloid progenitor cells at the promyelocytes/myelocyte stage $[1,2]$. In most $\mathrm{CN}$ patients, this granulocyte differentiation defect can be successfully treated by daily subcutaneous administration of recombinant human granulocyte colony-stimulating factor (G-CSF). Approximately $15 \%$ of $\mathrm{CN}$ patients develop myelodysplastic syndrome or acute myeloid leukemia (MDS or AML). Inherited mutations in the ELANE, HAX1, G6PC3, SRP54, GFI1, and JAGN1 genes cause CN, and leukemic progression has been seen in $\mathrm{CN}$ patients of all genetic groups [1].

$C S F 3 R$ mutations resulting in the production of truncated G-CSFR proteins that lack from one to four phospho-tyrosine residues and exhibit defective receptor 
internalization were reported in a majority of $\mathrm{CN}$ patients with overt AML or MDS [3-9]. However, transgenic d715 Csf $3 r$ mice lacking three tyrosines do not develop AML or MDS [3-9], suggesting that additional genetic alterations in combination with $C S F 3 R$ mutation are needed for the progression of AML. We recently examined a large cohort of CN/AML patients (31 patients) and found cooperative acquired mutations of $C S F 3 R$ and RUNX1 (runt-related transcription factor 1) in 55\% of CN patients with overt AML or MDS [10]. However, the detailed mechanism underlying the leukemogenic transformation downstream of $C S F 3 R$ and $R U N X 1$ mutations remained unknown.

Acquired mutations in RUNX1 occur in AML, mostly secondary to MDS, radiation therapy, or chemotherapy [11-16]. Most RUNX1 mutations are acquired heterozygous point mutations; they are predominantly located in the Runt homology/DNA-binding (RHD) or transactivation (TAD) domains. Interestingly, a majority of patients with familial platelet disorder (FPD) and a predisposition for AML have germline RUNX1 mutations [17]. Some FPD patients with overt AML gain additional RUNX1 mutations [17]. Among the described groups of AML patients, the incidence of acquired $R U N X 1$ mutations is the highest in CN/AML patients. RUNX1 mutations in CN/AML patients are distributed throughout the RHD (primarily) and TAD of the RUNX1 protein, and some hot spot positions have been noted [10]. For example, amino acid residues 139 and 174 of the RUNX1 protein were found to be mutated in four and three CN/AML patients, respectively [10] (data not shown). The functional outcomes of RUNX1 mutations at different positions have not yet been clearly defined, but we speculate that they may affect the DNA binding of RUNX1 to target genes or the protein-protein interactions, intracellular localization, protein stability, and/or post-translational modification(s) of RUNX1.

The role of inflammation in cancer was first mentioned in 1863 by Virchow [18]. A growing body of research suggests that pro-inflammatory signaling acts through diverse mechanisms to increase the proliferation rate of hematopoietic stem and progenitor cells (HSPCs), which induces genotoxicity, increases survival, and produces preleukemia stem cells (pre-LSCs) with a high likelihood of leukemic transformation [19].

In the present study, we sought to establish an in vitro experimental model to study the intracellular mechanisms of leukemia development downstream of CSF3R and RUNX1 mutations. Using this model, we identified upregulation of an inflammatory signature signaling in mouse HSPCs expressing mutated CSF $3 R$ and $R U N X 1$. This expression signature may predispose $\mathrm{CN}$ patients toward leukemic transformation.

\section{Material and methods}

\section{Mice}

Male d715 Csf $3 r$ mice on the C57BL/6J background have been described previously [9]. Mice were housed under pathogen-free conditions in the animal facility of Tübingen University.

\section{Cell purification and separation}

Mouse bone marrow cells were isolated by flushing the long bones with ice-cold PBS. Bone marrow mononuclear cells were isolated by Ficoll-Hypaque gradient centrifugation (Amersham Biosciences) and positively selected bone marrow lin $^{-}$cells by immunomagnetic labeling with corresponding MACS beads (Miltenyi Biotec). Cells were counted, and viability was assessed by Trypan blue dye exclusion.

\section{Generation of the lentiviral vectors expressing WT or mutant RUNX1 CDNA}

To generate RUNX1 mutants, we performed site-directed mutagenesis. As a template, we used Lego-iG/Puro-RUNX1-wtCTAP plasmid expressing human wild type (WT) RUNX1 generously provided by Dr. Boris Fehse and Dr. Carol Stocking. Specific primers to introduce mutations p.Arg139Gly (R139G) and p.Arg174Leu (R174L) in wild type RUNX1 nucleotide sequence were designed using the QuickChange Primer Design tool (https://www.agilent.com/store/primerDesignProgram.jsp). Primer sequences are available upon request. Lego-iG/PuroRUNX1-R139G-CTAP and Lego-iG/Puro-RUNX1-R174LCTAP plasmids were generated using the QuickChange II Site-Directed Mutagenesis Kit (Agilent Technologies, Inc.) according to the manufacturer's instruction. RUNXI cDNAs (WT and two mutants) were subsequently re-cloned into lentiviral pRRL.PPT.CBX3.SFFV.hRUNX1.i2.EBFP.puro.pre vector. Transgene expression was controlled by the spleen focusforming virus promoter (SFFV) juxtaposed to the minimal ubiquitous chromatin opening element (CBX3). EBFP translation was initiated by an internal ribosomal entry side (i2).

\section{Transduction of cells}

Lin $^{-}$cells were cultured in a hematopoietic stem cell expansion medium consisting of Stemline II medium supplemented with Pen/Strep, 10\% FCS (Sigma-Aldrich), $1 \mu \mathrm{m}$ dexamethasone, $100 \mathrm{ng} / \mathrm{ml}$ of $\mathrm{mSCF}, 4 \mathrm{ng} / \mathrm{ml}$ of $\mathrm{mIL}-3,10 \mathrm{ng} / \mathrm{ml}$ of $\mathrm{hIL}-6,40 \mathrm{ng} / \mathrm{ml}$ of murine IGF-1, and $20 \mathrm{ng} / \mathrm{ml}$ of human Flt$3 \mathrm{~L}$ at $2 \times 10^{5}$ cells $/ \mathrm{ml}$ for 2 days. Cells were transduced at MOIs of 5-10 in the presence of polybrene $(5 \mu \mathrm{g} / \mathrm{ml})$ and re-transduced after 12-24 h. Percentage of $\mathrm{EBFP}^{+}$cells was assessed by FACS. 
Detection of the human RUNX1 protein in transduced bone marrow lin ${ }^{-}$cells from d715 Csf3r mice using western blotting

A total of $1 \times 10^{6}$ transduced bone marrow lin $^{-}$cells from d715 Csf3r mice were lysed in $200 \mu \mathrm{l} 3 \times$ Laemmli buffer, and protein was denatured for $10 \mathrm{~min}$ at $95^{\circ} \mathrm{C}$. Five microliter of cell lysate in Laemmli buffer was loaded per lane. Proteins were separated on a $12.5 \%$ polyacrylamide gel and transferred on a nitrocellulose membrane (GE Healthcare) $(1 \mathrm{~h}, 100 \mathrm{~V}$, $4{ }^{\circ} \mathrm{C}$ ). The membrane was blocked for $1 \mathrm{~h}$ in $5 \%$ milk and incubated with primary rabbit anti-human and anti-mouse RUNX1-specific (Cell Signaling Technology \#4334) or GAPDH (Cell Signaling Technology, \#2118) antibody overnight (at $4{ }^{\circ} \mathrm{C}$ ). After that, membranes were washed and incubated with secondary HRP-conjugated antibody (Cell signaling, \#7074) for $1 \mathrm{~h}$ at room temperature. Pierce ECL solution (Thermo Fisher) and Amersham Hyperfilms were used to detect chemiluminescence signal of proteins.

\section{Liquid culture differentiation of transduced $\operatorname{lin}^{-}$cells}

A total of $2 \times 10^{5}$ of transduced cells $/ \mathrm{ml}$ were incubated for 7 days in RPMI 1640 GlutaMAX supplemented with $10 \%$ FCS, $10 \mathrm{ng} / \mathrm{ml} \mathrm{hIL-6,} 5 \mathrm{ng} / \mathrm{ml} \mathrm{IL-3,} 5 \mathrm{ng} / \mathrm{ml} \mathrm{GM-CSF}$, and $10 \mathrm{ng} / \mathrm{ml}$ G-CSF. The medium was exchanged every second day. On day 7, the medium was changed to RPMI 1640 GlutaMAX supplemented with $10 \%$ FBS, $1 \%$ penicillin/streptomycin, and $10 \mathrm{ng} / \mathrm{ml} \mathrm{G-CSF}$. The medium was exchanged every second day until day 11 . On day 11 , cells were analyzed by FACS using the following antibody: rat anti-mouse Gr-1 (BD 553128) and rat anti-mouse CD11b (BD 553312) on FACSCanto II.

\section{Colony-forming unit assay}

A total of 1000 transduced $\mathrm{EBFP}^{+}$cells were plated directly after transduction in $1 \mathrm{ml}$ methylcellulose medium (MethoCult GF M3434; StemCell Technologies) supplemented with $10 \mathrm{ng} / \mathrm{ml}$ of G-CSF. After 14 days of culture, the numbers of CFU-G, CFU-GM, and BFU-E colonies were counted. Cells were collected for the colony replating experiments, washed 3 times with PBS, and plated 1000 cells/dish in new methylcellulose for an additional 2 weeks (1st replating). The procedure was repeated one more time (2nd replating).

\section{Microarray-based mRNA expression analysis}

After $48 \mathrm{~h}$ of lentiviral transduction, lin $^{-}$cells were starved for $24 \mathrm{~h}$ and subsequently treated with $10 \mathrm{ng} / \mathrm{ml}$ of G-CSF for $24 \mathrm{~h}$. After that, transduced cells were sorted, and mRNA was isolated using the RNeasy Mini Kit (Qiagen, \#74106) according to the manufacturer's instructions.

RNA from transduced lin $^{-}$cells was subjected to microarray analysis using the Affymetrix Microarray Platform. The GeneChip WT cDNA Synthesis and Amplification Kit was used to make double-stranded cDNA from total RNA, which was then labeled with biotin (GeneChip WT Terminal Labeling Kit). After chemical fragmentation of the biotinlabeled cDNA targets, they were hybridized to the GeneChip Mouse Gene 2.0 ST Array using the Fluidics Station 450 and scanned using the Affymetrix GeneChip Scanner 3000 with the GeneChip Operating Software 1.4 (Affymetrix, Santa Clara, CA). Data analysis was performed using Affymetrix Expression Console Version 1.1 for invariant set normalization, and the Ingenuity Pathway Analysis (IPA) software (Qiagen) was used for identification of differentially expressed genes. Motif activity response analysis (MARA) was conducted using the Integrated System for Motif Activity Response Analysis (ISMARA); CEL files were uploaded to the server using the web interface.

\section{Cytospin preparation, staining, and microscopic image acquisition}

After sorting, $1 \times 10^{4}$ cells were centrifuge onto microscope slides at $250 \mathrm{rpm}$ for $3 \mathrm{~min}$. The slides were air-dried, and subsequent Wright-Giemsa staining was carried out. Images where acquired on a Nikon Eclipse TS100. Cells were covered with oil, and images were collected at $\times 630$ magnification.

\section{qRT-PCR}

RNA isolation was performed using the RNeasy Micro Kit (Qiagen). cDNA was synthesized from $1 \mu \mathrm{g}$ total RNA with the Omniscript RT Kit (Qiagen). qRT-PCR was conducted with SYBR Green qPCR master mix (Roche) on a Light Cycler 480 (Roche). Target genes were normalized to ACTB. Primers are available upon request.

\section{LSK cell analysis}

A total of $2 \times 10^{5}$ cells were incubated with FcR blocking antibody (BioLegend \#101320), 7AAD, and lineage cocktail biotin-conjugated antibody (BioLegend \#79750, \#79748, \#79752, \#79748, \#79749), and stained with APC-Cy7conjugated streptavidin (BioLegend \#405208), anti-mouse Sca-1 BV510-conjugated antibody (BioLegend, \#108129), and anti-mouse-c-KIT APC-conjugated antibody (BioLegend, \#105812). After staining and washing, cells were analyzed on a CANTO II (BD) flow cytometer. 


\section{Statistics}

Statistical analysis was performed using a two-sided unpaired Student $t$ test for the analysis of differences in mean values between groups.

\section{Results}

\section{Diminished formation of myeloid colonies and elevated replating capacity of d715 Csf3r lin ${ }^{-}$cells transduced with mutated RUNX1}

We isolated bone marrow lin ${ }^{-}$cells from d 715 Csf $3 r$ mice and transduced these cells with lentiviral vectors encoding EBFP and RUNX1 wild type (RUNX1-WT), or mutants, RUNX1R139G, or RUNX1-R174L. Amino acids 174 and 139 were found to be "hot spots" for mutation in the RHD domain of the RUNX1 protein, as they were detected in four and three CNAML patients, respectively (Fig. 1a) [10] (data not shown). At $72 \mathrm{~h}$ post-transduction, we performed colony-forming units and replating experiments of sorted $\mathrm{EBFP}^{+}$cells (Fig. 1b). We observed comparable expression of human WT and mutant RUNX1 proteins in transduced cells (Fig. 1c). Low levels of endogenous murine runx1 protein were detected in $\mathrm{BFP}^{+}$ control transduced cells (Fig. 1c). Interestingly, d715 Csf $3 r$ lin $^{-}$cells transduced with mutated RUNX1 had markedly diminished capacities to form myeloid colonies, including CFU$\mathrm{G}$ and CFU-GM, as compared with cells transduced with WT RUNX1 (Fig. 1d). In line with these findings, CFU replating experiments showed that cell transduced with each RUNXI mutant had markedly higher replating capacities than RUNX1 WT-transduced cells (Fig. 1e).

\section{Reduced liquid culture myeloid differentiation of d715 Csf3r lin ${ }^{-}$cells transduced with mutated $R U N X 1$}

We next compared the G-CSF-triggered myeloid differentiation of transduced d715 Csf $3 r$ lin $^{-}$cells in vitro. Transduced cells were cultured in liquid culture myeloid differentiation medium for 11 days (Fig. 2a). FACS was used to count the absolute numbers of myeloid $\mathrm{CD} 11 \mathrm{~b}^{+}$and $\mathrm{Gr}-1^{+}$cells on day 11 of liquid culture, and the results showed that d715 Csf $3 r$ lin $^{-}$cells transduced with each of the RUNX1 mutants exhibited reduced myeloid differentiation, compared with WT $R U N X 1$-overexpressing samples (Fig. 2b). At the same time, no marked difference was observed in the absolute numbers of $\mathrm{BFP}^{+}$cells between groups transduced with WT RUNX1 or with each of RUNX1 mutants on days 3 and day 7 of liquid culture differentiation. On day 11 of culture, numbers of $\mathrm{BFP}^{+}$ cells transduced with $R U N X 1-\mathrm{R} 139 \mathrm{G}$ mutant were significantly $(p<0.05)$ increased, as compared with RUNX1 WTor $R U N X 1-\mathrm{R} 174 \mathrm{~L}-$ transduced samples (Fig. 2c).
RUNX1 missense mutations induce inflammatory signaling pathways in G-CSF-treated d715 Csf3r hematopoietic cells

We further aimed to identify intracellular signaling pathways downstream of csf3r and RUNX1 mutations that may affect the in vitro proliferation and myeloid differentiation of mouse HSPCs. The d715 Csf $3 r$ lin $^{-}$cells were expanded in HSPC expansion medium and transduced with lentiviral vectors carrying RUNX1 WT, RUNX1-R139G, or RUNX1-R174L. Transduced cells were starved for $24 \mathrm{~h}$ and then treated with G-CSF for $24 \mathrm{~h}$. EBFP ${ }^{+}$cells were sorted in RLT buffer, and mRNA expression was evaluated using an Affymetrix MoGene 2.0 Chip (Fig. 3a). Representative images of Wright-Giemsa-stained cytospins prepared from sorted cells show no difference in the cell morphology between studied groups: all samples show immature cell morphology (Fig. 3b). These data suggest that the differences in mRNA expression should not contribute to a strong diversity in the cell composition between studied groups.

After invariant set normalization, the expression levels of the RUNX1 mutants were normalized to those obtained from WT RUNX1-transduced cells. The fold change expression table (Suppl. Table 1) was uploaded to the IPA software, and the cutoff values were set to -1.7 and +1.7 for log fold change, with the goal of identifying differentially expressed genes. This analysis showed that 1113 and 1814 genes were differentially expressed in the RUNX1-R174L and RUNX1R139G mutant groups compared with the WT RUNX1 group (Suppl. Table 2). Overlap analysis revealed that 679 genes (37.4\% for the RUNX1-R139G mutant and $61 \%$ for the RUNX1-R174L mutant) were co-regulated in both RUNX1 mutant groups (Fig. 3c). The 15 most highly up- and downregulated genes were very similar between the $R U N X 1$ mutant groups. Of the top upregulated genes, 10/15 from the RUNXI$\mathrm{R} 174 \mathrm{~L}$ group and 14/15 from the $R U N X 1-\mathrm{R} 139 \mathrm{G}$ group were co-activated in both groups. Of the top downregulated genes, $14 / 15$ from the $R U N X 1-\mathrm{R} 174 \mathrm{~L}$ group and 11/15 from the $R U N X 1-\mathrm{R} 139 \mathrm{G}$ group were co-downregulated (Fig. 3d). A comparison analysis performed using the IPA software revealed that 46 and 79 canonical pathways were significantly enriched $(\log (p$ value $) \geq 1.3$ corresponding to $p \leq 0.05)$ in d715 Csf $3 r$ lin $^{-}$cells transduced with $R U N X 1-\mathrm{R} 174 \mathrm{~L}$ or $R U N X 1-\mathrm{R} 139 \mathrm{G}$, respectively. Of these pathways, 11 in the $R U N X 1-\mathrm{R} 174 \mathrm{~L}$ group and 18 in the $R U N X 1-\mathrm{R} 139 \mathrm{G}$ group were significantly activated $(z$-value $\geq 2$ ) or inhibited ( $z$-value $\leq-2$ ). Most of the regulated pathways belonged to members of the activated innate immune pathways category; these included NF-kappaB signaling, toll-like receptor signaling (TLR), acute-phase response signaling, production of nitric oxide and reactive oxygen species, pattern-recognizing receptors, Trem 1 signaling, and IL-1 signaling. Activation was also seen among members of the inflammatory signaling 
Fig. 1 In HSPCs of d 715 Csf3r mice, $R U N X 1$ mutations decrease CFU-G and CFU-GM formation but increase the replating capacity. a Schematic of RUNX1 protein showing the location and amino acid changes of the mutations, which are indicated by black triangles. The functionally important Runt homology DNAbinding domain (RHD) is shown in blue, and the transactivation domain (TAD) is shown in red. b Schematic of CFU experiments performed using transduced bone marrow lin ${ }^{-}$cells of C57BL/6d715 Csf $3 r$ mice. c

Representative WB images of lin cells of C57BL/6-d715 Csf3r mice transduced with corresponding lentiviral constructs. GAPDH was used as loading control. d CFU assay and e replating CFU assay of transduced C57BL/6-d715 Csf3r $\operatorname{lin}^{-}$cells. Data represent means \pm SD from triplicates of two independent experiments; $* p<0.05, * * p<0.01$,*** $p<0.001$ a

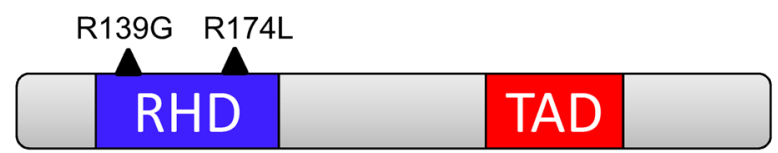

b
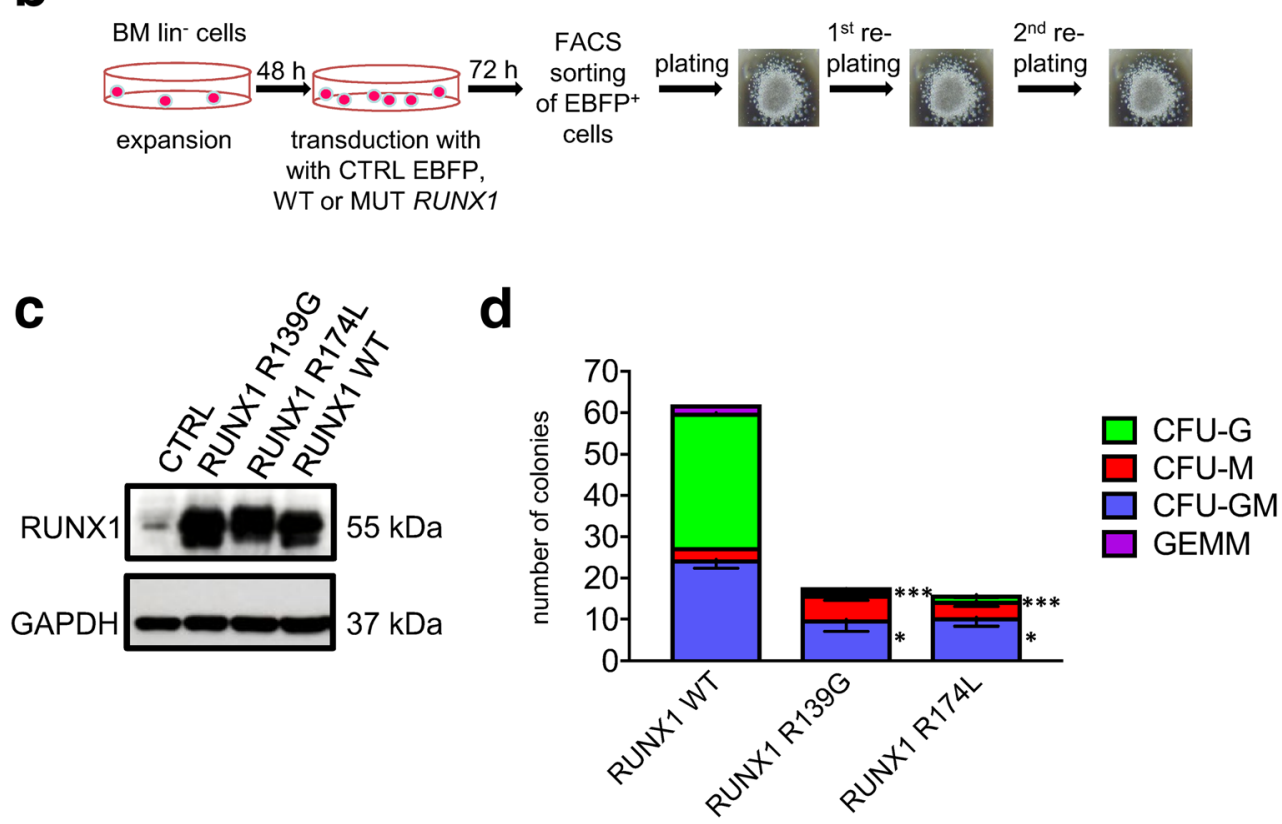

e

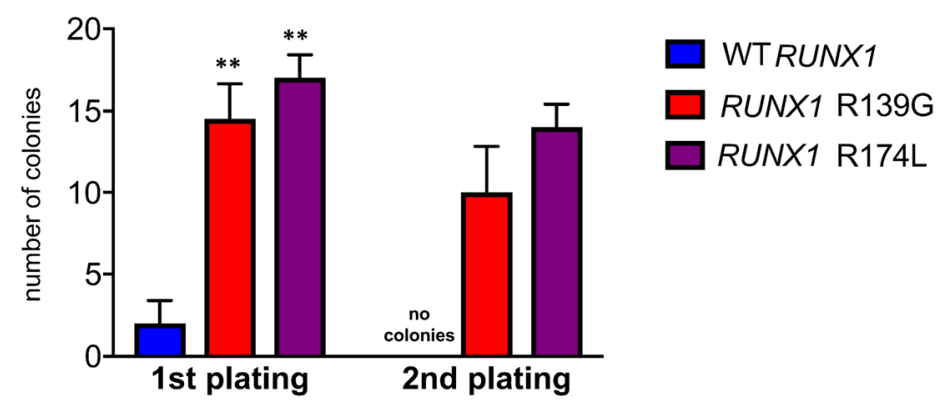

pathways, such as IL-6 signaling, interferon signaling, Tec kinase signaling, and leukocyte extravasation signaling (Fig. 3e, Suppl. Table 3).

\section{Representative pathways of the innate immune system activated in $\mathrm{d} 715 \mathrm{Csf3r} \mathrm{lin}^{-}$cells transduced with RUNX1 mutants}

As examples of the activated canonical inflammatory and innate immune signaling pathways, canonical TLR signaling and IL-6 pathways are depicted in Suppl. Figs. 2 and 3. TLR signaling was upregulated in d715 Csf3r lin cells transduced with the R139G or R174L RUNX1 mutants; this resulted in activation of transcription factors through Janus kinase 1 (JAK1) and p38 MAPK, promoting the expression of IL-1, TNF-alpha, and IL12 (Suppl. Fig. 2A, B). Interferon signaling acted through JAK1 and JAK2 to activate the transcription factors, STAT1 and STAT2, thereby upregulating interferon response factors and other pro-inflammatory genes (Suppl. Fig. 2A, B).

The upregulation of IL-6 signaling-dependent genes in d715 Csf $3 r$ lin $^{-}$cells transduced with each RUNX1 mutant was also mediated through JAK signaling, but relied on the transcription factor, STAT3, to confer translational regulation in the nucleus. Additional IL-6-dependent activation of ERK1 induced the NF-kappaB-NF-IL-6 transcription factor complex (Suppl. Fig. 3A, B). 
a

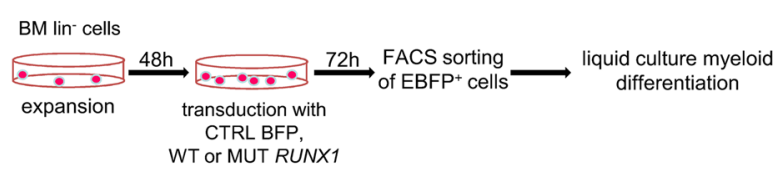

b

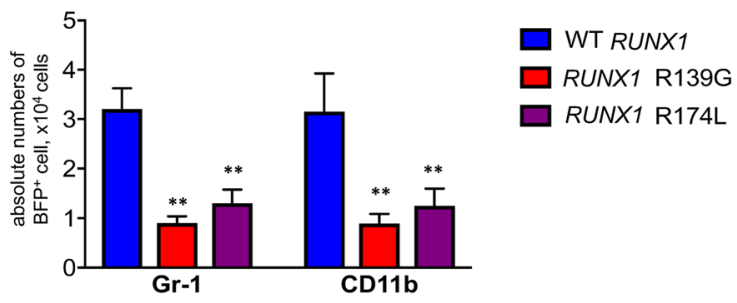

C

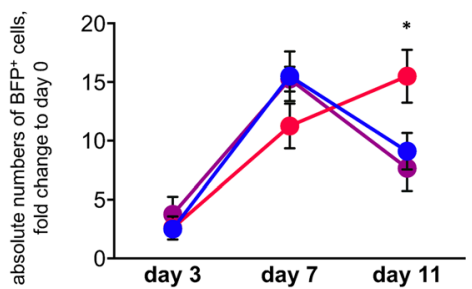

Fig. 2 Liquid culture differentiation of transduced d715 Cs $3 r$ HSPCs. a Schematic of the workflow for liquid culture myeloid differentiation of transduced bone marrow lin ${ }^{-}$cells obtained from C57BL/6-d715 Csf3r mice. b Transduced cells were subjected to liquid culture myeloid differentiation (see "Material and methods" for details). FACS was used to count the myeloid and granulocytic cells on day 11 of culture. Graph bars represent absolute cell counts of $\mathrm{Gr}-1^{+}$or $\mathrm{CD} 11 \mathrm{~b}^{+}$cells. Data represent means \pm SD from triplicates of two independent experiments; $* * p<0.01$

\section{Upstream pathway analysis points toward inflammatory dysregulation}

Upstream analysis performed using the IPA software identified potential upstream regulators responsible for the gene expression signatures observed in the studied groups. We detected 768 upstream regulators in the RUNX1-R139G mutant group and 875 regulators in the $R U N X 1$-R174L mutant group (Suppl. Fig. 4A, Suppl. Table 4). Five hundred upstream regulators were commonly found in both groups: $65.1 \%$ in the RUNX1-R139G mutant group and $57.1 \%$ in the RUNX1R174L mutant group (Suppl. Fig. 4A). Among the 15 top activated upstream regulators, $9 / 15$ for $R U N X 1-\mathrm{R} 174 \mathrm{~L}$ and 11/15 for RUNX1-R139G were shared between the groups. Among the 15 top downregulated regulators, overlaps were seen in 6/15 for RUNX1-R174L and 9/15 for RUNX1-R139G (Suppl. Fig. 4B). Selected up- and downregulated candidate genes were validated by qRT-PCR in the independent set of experiments (Suppl. Fig. 4C). Also, staining of transduced GCSF-treated lin ${ }^{-}$cells with Sca-1 and c-kit antibody followed by flow cytometry analysis revealed an increase of $\operatorname{lin}^{-} \mathrm{Sca}-$ $1^{+} \mathrm{c}-\mathrm{KIT}^{+}$cell population in RUNX1-R139G- and RUNX1R174L-transduced groups, compared with cells expressing RUNX1 WT, or GFP CTRL (Suppl. Fig. 4D).

The top overlapping upstream regulators included a number of inflammatory cytokines, such as IL-15 and IL-18. IL-15 has been described as a responsible driver for chronic inflammation in autoimmune diseases and hematological malignancies [20]. Both IL-15 and IL-18 are confirmed targets for antitumor activity $[21,22]$. Another factor that promotes survival of leukemogenic cells, Bcl2a1 [23], was also highly expressed, as were Ly6a and Sca-1, which are known as stemness factors for HSPCs. ${ }^{24}$ The top downregulated genes included anti-inflammatory genes, such as Il-10 [25], and the cytokine-processing factor, Dpp4, which can increase proliferation by prolonging cytokine signaling [26]. Interestingly, $C s f 3 r$ was among the highest expressed genes in both groups. This is in accordance with the literature showing that G-CSF sensitivity is increased in AML blasts with missense RUNXI mutations.

\section{Motif activity response analysis confirms the central role of inflammatory and innate immunity signaling and the presence of early molecular changes related to MDS/AML downstream of Csf3r and RUNX1 mutations}

We used the ISMARA [27] web tool to analyze the transcription factor binding motifs enriched among the differentially expressed genes. We found that the most highly enriched motif was Irf2_Irf1_Irf8_Irf9_Irf7 (z-value 4.564), which corresponds to the interferon regulatory factors. This indicates that an interferon-related change in inflammation signaling is responsible for at least some of the observed gene expression differences. The Stat 2 motif was significantly correlated with the expression signature ( $z$-value 2.707), and we observed significant activation of the Klf4_Sp3 ( $z$-value 2.349) and Mecp-2 (z-value 2.627) transcription factor binding motifs known to be associated with AML or MDS, but significant downregulation of the Max-Mycn motif (z-value 2.016), upon transduction of d715 Csf $3 r$ lin $^{-}$cells with $R U N X 1$ mutants (Suppl. Fig. 5A). Finally, our inferred activity analysis of each motif revealed that there was a high degree of similarity in the significantly activated motifs associated with the two different RUNX1 mutants (Suppl. Fig. 5B).

\section{Discussion}

The identification of cooperative CSF3R and RUNX1 mutations in a majority of $\mathrm{CN}$ patients with overt MDS or AML brought us one step closer to understanding leukemia development [3]. Truncated CSF3R mutations are a very rare event 
Fig. 3 Canonical pathway analysis of microarray data obtained from transduced d715 $C s f 3 r$ HSPCs. a Schematic of the experimental procedure performed for microarray analysis. The experiment was conducted in duplicate. After quality control analysis, one sample from the RUNX1-R139G group was excluded from the final analysis. b Wright-Giemsastained cytospin preparations of $C s f 3 r$ lin $^{-}$cells transduced with RUNXI WT and mutants and sorted for fluorescent protein expression. Images were acquired at $\times 630$ magnification. $\mathrm{c}$ Venn diagram depicting the overlay of significantly up- or

downregulated transcripts in each $R U N X 1$ mutant group, as compared with WT RUNX1transduced cells. d Canonical pathways that were significantly $(-\log (p$ value $)>1.3)$ enriched and significantly predicted $(z-$ value $>2$ and $<-2$ ) to be up- or downregulated in each RUNXI mutant group compared with WT RUNX1-transduced cells. Shared pathways are marked with an asterisk (*). e IPA analysis of the significantly regulated pathways shared by lin $^{-}$cells transduced with each RUNXI mutant, as compared with WT RUNX1overexpressing samples. Upregulated pathways are shown in orange, and downregulated pathways are shown in blue

a

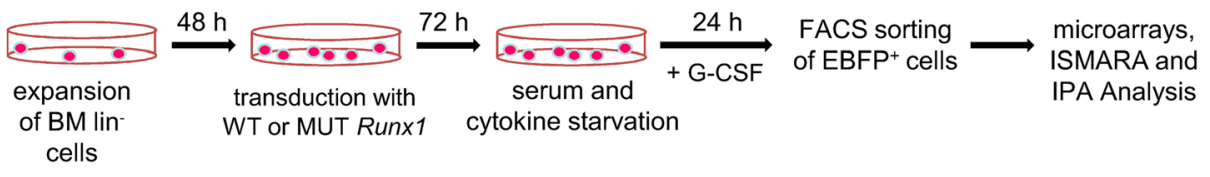

b
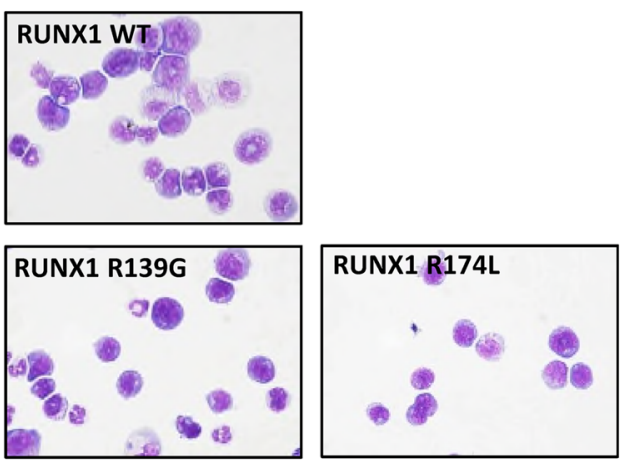

C
vS WT RUNX1

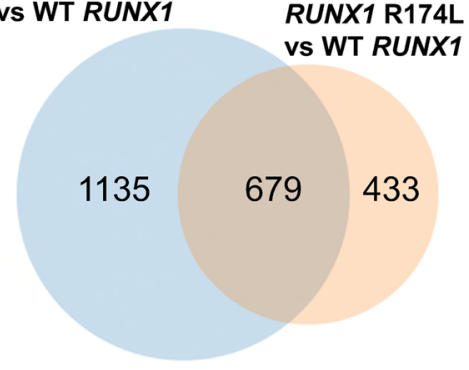

d

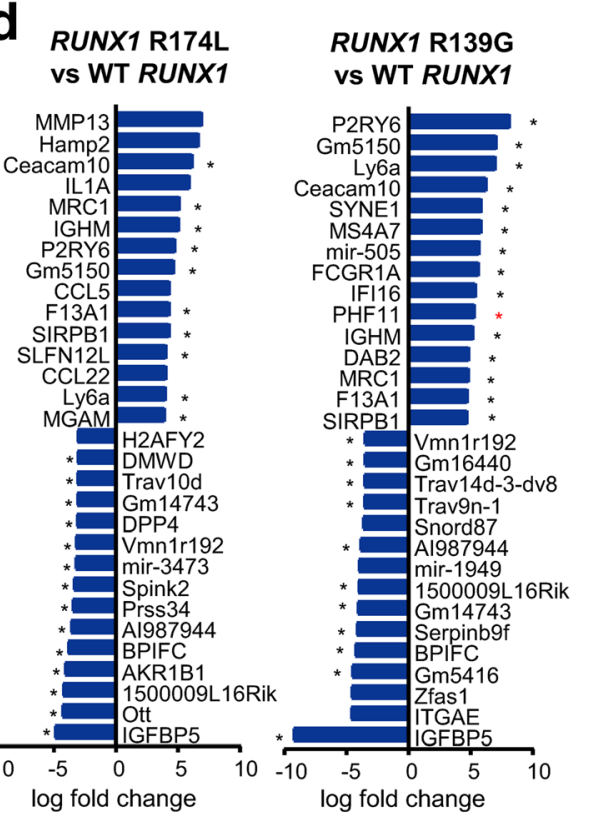

e

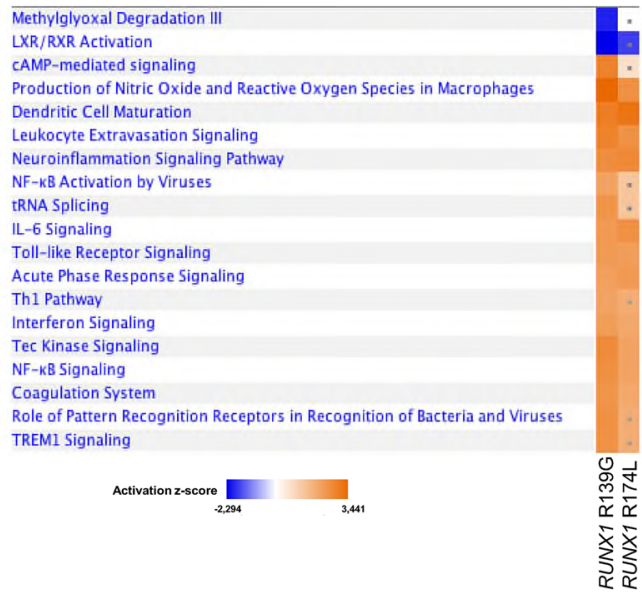

Figure 3 in de novo AML, while RUNX1 mutations are most frequent among patients with secondary AML (sAML) after chemotherapy or radiation therapy, which represents about $30 \%$ of adult AML cases. Acquired cooperative CSF3R and RUNX1 mutations in approximately $55 \%$ of CN/AML patients are a unique feature and may reflect the inherited background of patients with $\mathrm{CN}$-associated gene mutations, such as those in ELANE or HAX1. Mechanistically, ELANE mutations induce unfolded protein response (UPR) [28, 29] and endoplasmic reticulum (ER) stress, while mutations in $H A X 1$ have proapoptotic functions [30,31]. The role of inherited $\mathrm{CN}$ - associated mutations in the process of leukemogenesis needs to be investigated. The presence of these mutations may predispose to acquisition of secondary mutations in CSF3R and $R U N X 1$ and the development of leukemia. Similar to secondary leukemias, wherein HSPCs are damaged by chemotherapeutic agents or radiation therapy, inherited $\mathrm{CN}$-specific mutations may induce DNA damage or stress responses in HSPCs. Indeed, using CN patient-derived iPSCs as an experimental model, we recently showed that an elevation of DNA damage in CN HSPCs precedes the leukemic transformation [32]. In the present study, we found that $c s f 3 r$ and $R U N X 1$ 
Fig. 4 Proposed model for leukemia development in $\mathrm{CN}$. a $\mathrm{CN}, \mathrm{CN}$-associated germline mutations cause maturation arrest of granulopoiesis at the stage of promyelocytes/myelocyte. $\mathrm{CN}$ CHIP, HSPCs that acquire CSF3R mutations gain a proliferative advantage that may mimic the CHIP phenotype. CN pre-leukemia, the co-acquisition of RUNX1 mutation induces an inflammatory milieu, leading to genotoxicity, additional defects of myeloid differentiation, and elevated proliferation that constitute the pre-leukemia stage. CN/AML, the acquisition of additional leukemia-associated gene mutations or chromosomal abnormalities results in AML or MDS a

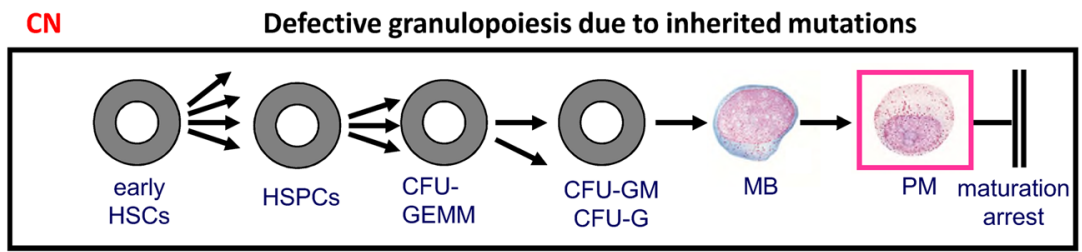

CN CHIP Acquisition of CSF3R mutations, proliferative advantage of HSPCs, CHIP

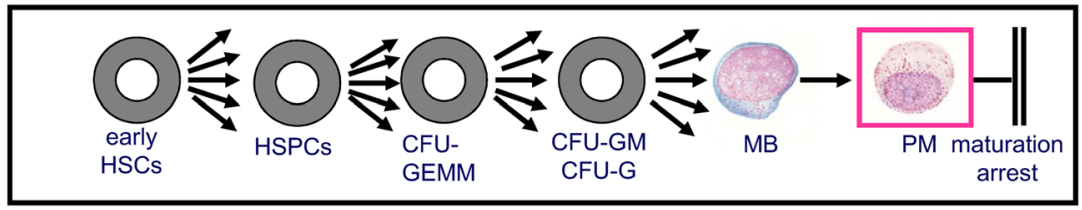

CSF3R mutations, co-acquisition of RUNX1 mutations, CN pre-leukemia proinflammatory mileu, epigenetic remodeling
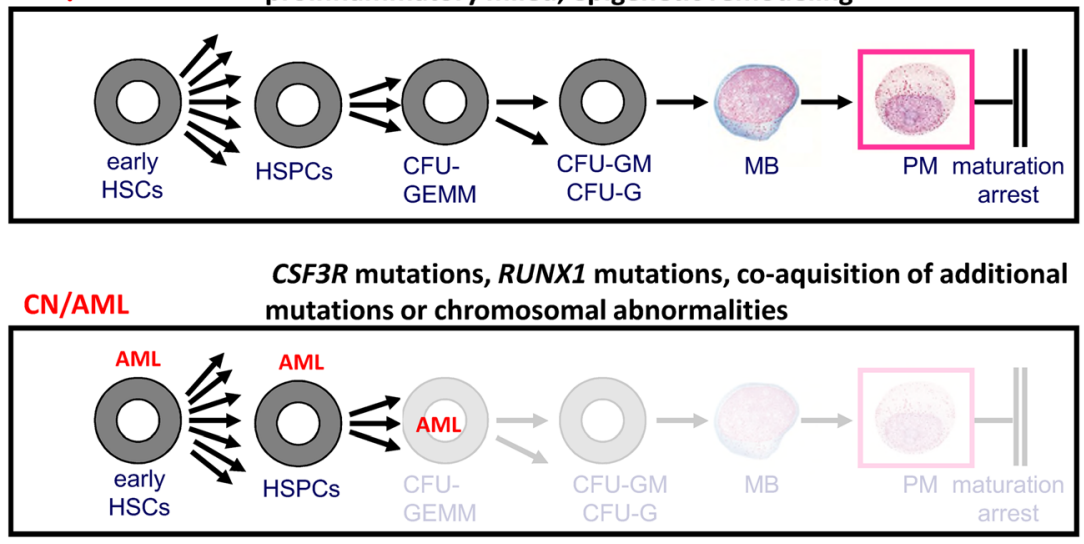

mutations in HSPCs induce reduced myeloid differentiation and enhanced clonogenic potential. Our findings demonstrated that cells transduced with WT RUNX1 differentiated and lost the capacity to proliferate, making no colonies in replating experiments. In contrast, cells transduced with RUNX1 mutants differentiate less, but retain proliferative potential. Leukemogenic activity of $\operatorname{csf} 3 R$ and $R U N X 1$ mutations should be further elaborated in vivo using a mouse model.

Elucidation of the deregulated intracellular signaling pathways downstream of CSF3R and RUNX1 mutations will help to identify druggable targets, with the goal of eliminating leukemia-predisposed HSPCs and/or specifically targeting $\mathrm{CN} / \mathrm{AML}$ blasts. Interestingly, mRNA expression analysis revealed that HSPCs harboring mutations in Csf3R and RUNX1 exhibit activation of the inflammatory and innate immunity pathways, including interferon signaling; IL-1, IL-6, IL-8, and TLR signaling; and TREM1 signaling. Additionally, we also found marked upregulation of Ly6a (also known as Sca-1), a marker of stemness in HSPCs [24] that is also upregulated upon inflammation [33-35]. IL-6 and IL-8 were previously known to be hyper-activated in MDS and AML [19, 36, 37]. Moreover, the increased genotoxic stress in HSPCs of $40 \%$ de novo MDS cases has been associated with elevated TLRs/Myd88-triggered intracellular signaling and IL-8 expression [19]. Most probably, the presence of truncated CSF3R and RUNX1 mutations in HSPCs of $\mathrm{CN}$ patients alters the pro-inflammatory cell state, enhances proliferation, and increases the susceptibility of HSPCs to genomic toxicity. Recent reports revealed that preleukemic HSPCs carrying an ETV6-RUNX1 fusion gene or having Pax5 haploinsufficiency evolved to precursor B cell acute lymphocytic leukemia upon activation of the proinflammatory pathways $[38,39]$. The role of activated innate immunity and inflammatory pathways in the leukemogenesis of CN HSPCs was not studied yet. It would be interesting to investigate the susceptibility and expression kinetics of TLRs and the receptors for IL-1 and IL-8 on HSPCs from CN/AML patients during the development of leukemia.

We found that the Spil/PU.1 transcription factor motif is activated in HSPCs downstream of Csf3r and RUNX1 mutations. PU.1 is upregulated in hematopoietic cells of $\mathrm{CN}$ patients [40] and is an essential transcription factor for monocytic differentiation [41, 42]. PU.1 also acts as a maintenance factor for pre- and leukemia-initiating cells [43]. The 
leukemogenic function of upregulated PU.1 in HSPCs of CN patients has not been studied yet.

Taken together, our results support the following proposed mechanism for leukemia development in CN: CSF3R mutations represent a state of clonal hematopoiesis of indeterminate potential (CHIP) in CN patients in that they confer a clonal advantage. Co-acquisition of RUNX1 mutation further increases proliferation and genotoxicity, including hypersensitivity to proinflammatory signaling. This combination may lead to acquisition of additional somatic mutations (e.g., in SUZ12 or ASXL1) or chromosomal abnormalities (e.g., monosomy 7 or trisomy 21), which finally overt to MDS or AML (Fig. 4).

Acknowledgments We would like to thank C. Stocking for providing $R U N X 1$ expression plasmids.

Authorship contributions JS and MR made initial observations, designed the experiments, analyzed the data, supervised experimentation, and wrote the manuscript; MR performed the main experiments; MK generated RUNX1 mutants; A Scham. and DH re-cloned RUNX1 cDNAs into pRRL.PPT.CBX3SF hRUNX1.i2.EBFP.puro.pre backbone; OK assisted with the transduction of mouse lin $^{-}$cells and differentiation experiments; A Schm. and DCL provided bone marrow of C57BL/6-d715 Csf $3 r$ mice; and $\mathrm{KW}$ and LK provided insightful comments.

Funding information Open Access funding provided by Projekt DEAL. This study was funded by the Fritz Thyssen Stiftung and DFG. We would like to acknowledge the financial support of the study by the German Jose Carreras Leukemia foundation (M.R. and J.S.).

\section{Compliance with ethical standards}

Conflict of interest The authors declare that they have no conflict of interest.

Ethical approval All applicable international, national, and/or institutional guidelines for the care and use of animals were followed. All animal studies included in this manuscript were reviewed and approved by the Regional Board of the City of Tübingen.

Human and animal rights This article does not contain any study with humans participants performed by any of the authors.

Open Access This article is licensed under a Creative Commons Attribution 4.0 International License, which permits use, sharing, adaptation, distribution and reproduction in any medium or format, as long as you give appropriate credit to the original author(s) and the source, provide a link to the Creative Commons licence, and indicate if changes were made. The images or other third party material in this article are included in the article's Creative Commons licence, unless indicated otherwise in a credit line to the material. If material is not included in the article's Creative Commons licence and your intended use is not permitted by statutory regulation or exceeds the permitted use, you will need to obtain permission directly from the copyright holder. To view a copy of this licence, visit http://creativecommons.org/licenses/by/4.0/.

\section{References}

1. Skokowa J, Dale DC, Touw IP, Zeidler C, Welte K (2017) Severe congenital neutropenias. Nat Rev Dis Prim 3:17032
2. Skokowa J, Germeshausen M, Zeidler C et al (2007) Severe congenital neutropenia: inheritance and pathophysiology. Curr Opin Hematol 14:22-28

3. Hermans MHA, Antonissen C, Ward AC, Mayen AEM, Ploemacher RE, Touw IP (1999) Sustained receptor activation and hyperproliferation in response to granulocyte colonystimulating factor (G-CSF) in mice with a severe congenital neutropenia/acute myeloid leukemia-derived mutation in the GCSF receptor gene. J Exp Med 189:683-691

4. Dong F, Brynes RK, Tidow N, Welte K, Löwenberg B, Touw IP (1995) Mutations in the gene for the granulocyte colonystimulating-factor receptor in patients with acute myeloid leukemia preceded by severe congenital neutropenia. N Engl J Med 333:487493

5. Dong F, Hoefsloot LH, Schelen AM, Broeders CA, Meijer Y, Veerman AJ, Touw IP, Lowenberg B (1994) Identification of a nonsense mutation in the granulocyte-colony-stimulating factor receptor in severe congenital neutropenia. Proc Natl Acad Sci 91: 4480-4484

6. Dong F, van Buitenen C, Pouwels K, Hoefsloot LH, Löwenberg B, Touw IP (1993) Distinct cytoplasmic regions of the human granulocyte colony-stimulating factor receptor involved in induction of proliferation and maturation. Mol Cell Biol 13:7774-7781

7. Ward AC, van Aesch YM, Schelen AM, Touw IP (1999) Defective internalization and sustained activation of truncated granulocyte colony-stimulating factor receptor found in severe congenital neutropenia/acute myeloid leukemia. Blood 93:447-458

8. Liu F, Kunter G, Krem MM, Eades WC, Cain JA, Tomasson MH, Hennighausen L, Link DC (2008) Csf3r mutations in mice confer a strong clonal HSC advantage via activation of Stat5. J Clin Invest 118:946-955

9. McLemore ML, Poursine-Laurent J, Link DC (1998) Increased granulocyte colony-stimulating factor responsiveness but normal resting granulopoiesis in mice carrying a targeted granulocyte colony-stimulating factor receptor mutation derived from a patient with severe congenital neutropenia. J Clin Invest 102:483-492

10. Skokowa J, Steinemann D, Katsman-Kuipers JE, Zeidler C, Klimenkova O, Klimiankou M, Ünalan M, Kandabarau S, Makaryan V, Beekman R, Behrens K, Stocking C, Obenauer J, Schnittger S, Kohlmann A, Valkhof MG, Hoogenboezem R, Göhring G, Reinhardt D, Schlegelberger B, Stanulla M, Vandenberghe P, Donadieu J, Zwaan CM, Touw IP, van den Heuvel-Eibrink MM, Dale DC, Welte K (2014) Cooperativity of RUNX1 and CSF3R mutations in severe congenital neutropenia: a unique pathway in myeloid leukemogenesis. Blood 123:2229 2237

11. Osato M (2004) Point mutations in the RUNX1/AML1 gene: another actor in RUNX leukemia. Oncogene 23:4284-4296

12. Osato M, Asou N, Abdalla E, Hoshino K, Yamasaki H, Okubo T, Suzushima H, Takatsuki K, Kanno T, Shigesada K, Ito Y (1999) Biallelic and heterozygous point mutations in the runt domain of the AML1/PEBP2alphaB gene associated with myeloblastic leukemias. Blood 93:1817-1824

13. Christiansen DH, Andersen MK, Pedersen-Bjergaard J (2004) Mutations of AML1 are common in therapy-related myelodysplasia following therapy with alkylating agents and are significantly associated with deletion or loss of chromosome arm 7q and with subsequent leukemic transformation. Blood 104:1474-1481

14. Harada H, Harada Y, Tanaka H, Kimura A, Inaba T (2003) Implications of somatic mutations in the AML1 gene in radiationassociated and therapy-related myelodysplastic syndrome/acute myeloid leukemia. Blood 101:673-680

15. Schnittger S, Dicker F, Kern W, Wendland N, Sundermann J, Alpermann T, Haferlach C, Haferlach T (2011) RUNX1 mutations are frequent in de novo-AML with noncomplex karyotype and confer an unfavorable prognosis. Blood 117:2348-2357 
16. Gaidzik VI, Teleanu V, Papaemmanuil E et al (2016) RUNX1 mutations in acute myeloid leukemia are associated with distinct clinico-pathologic and genetic features. Leukemia 30:2160-2168

17. Preudhomme C, Renneville A, Bourdon V et al (2009) Brief report High frequency of RUNX1 biallelic alteration in acute myeloid leukemia secondary to familial platelet disorder. 113:5583-5588

18. Balkwill F, Mantovani A (2001) Inflammation and cancer: back to Virchow? Lancet 357:539-545

19. Hemmati S, Haque T, Gritsman K (2017) Inflammatory signaling pathways in preleukemic and leukemic stem cells. Front Oncol 7

20. Fehniger TA, Caligiuri MA (2001) Interleukin 15: biology and relevance to human disease. Blood 97:14-32

21. Kim PS, Kwilas AR, Xu W et al (2016) IL-15 superagonist/IL$15 \mathrm{R} \alpha$ Sushi-Fc fusion complex (IL-15SA/IL-15R $\alpha \mathrm{Su}-\mathrm{Fc}$; ALT803) markedly enhances specific subpopulations of NK and memory $\mathrm{CD} 8+\mathrm{T}$ cells, and mediates potent anti-tumor activity against murine breast and colon carcinomas. Oncotarget 7

22. Senju H, Kumagai A, Nakamura Y, Yamaguchi H, Nakatomi K, Fukami S, Shiraishi K, Harada Y, Nakamura M, Okamura H, Tanaka Y, Mukae H (2018) Effect of IL-18 on the expansion and phenotype of human natural killer cells: application to cancer immunotherapy. Int J Biol Sci 14:331-340

23. Vogler M (2012) BCL2A1: the underdog in the BCL2 family. Cell Death Differ 19:67-74

24. Holmes C, Stanford WL (2007) Concise review: stem cell antigen1: expression, function, and enigma. Stem Cells 25:1339-1347

25. Kelly Á, Lynch A, Vereker E, Nolan Y, Queenan P, Whittaker E, O'Neill LAJ, Lynch MA (2001) The anti-inflammatory cytokine, interleukin (IL)-10, blocks the inhibitory effect of IL-1 $\beta$ on long term potentiation. J Biol Chem 276:45564-45572

26. Deng L, Chan R, O'Leary HA et al (2017) DPP4 truncated GMCSF and IL-3 manifest distinct receptor-binding and regulatory functions compared with their full-length forms. Leukemia 31: 2468-2478

27. Balwierz PJ, Pachkov M, Arnold P, Gruber AJ, Zavolan M, van Nimwegen E (2014) ISMARA: automated modeling of genomic signals as a democracy of regulatory motifs. Genome Res 24:869884

28. Grenda DS, Murakami M, Ghatak J, Xia J, Boxer LA, Dale D, Dinauer MC, Link DC (2007) Mutations of the ELA2 gene found in patients with severe congenital neutropenia induce the unfolded protein response and cellular apoptosis. Blood 110:4179-4187

29. Nustede R, Klimiankou M, Klimenkova O, Kuznetsova I, Zeidler C, Welte K, Skokowa J (2016) ELANE mutant-specific activation of different UPR pathways in congenital neutropenia. Br J Haematol 172:219-227

30. Boztug K, Appaswamy G, Ashikov A et al (2008) A syndrome with congenital neutropenia and mutations in G6PC3. N Engl J Med 360:32-43

31. Klein C, Grudzien M, Appaswamy G, Germeshausen M, Sandrock I, Schäffer AA, Rathinam C, Boztug K, Schwinzer B, Rezaei N, Bohn G, Melin M, Carlsson G, Fadeel B, Dahl N, Palmblad J, Henter JI, Zeidler C, Grimbacher B, Welte K (2007) HAX1 deficiency causes autosomal recessive severe congenital neutropenia (Kostmann disease). Nat Genet 39:86-92

32. Dannenmann B, Zahabi A, Mir P et al (2018) Human iPSC-based model of severe congenital neutropenia reveals elevated UPR and DNA damage in CD34+ cells preceding leukemic transformation. Exp Hematol

33. Clapes T, Lefkopoulos S, Trompouki E (2016) Stress and nonstress roles of inflammatory signals during HSC emergence and maintenance. Front Immunol 7:1-15

34. Essers MAG, Offner S, Blanco-Bose WE, Waibler Z, Kalinke U, Duchosal MA, Trumpp A (2009) IFN $\alpha$ activates dormant haematopoietic stem cells in vivo. Nature 458:904-908

35. Bujanover N, Goldstein O, Greenshpan Y, Turgeman H, Klainberger A, Scharff Y', Gazit R (2018) Identification of immune-activated hematopoietic stem cells. Leukemia 32:20162020

36. Kuett A, Rieger C, Perathoner D et al (2015) IL-8 as mediator in the microenvironment-leukaemia network in acute myeloid leukaemia. Sci Rep 5:1-11

37. Bhattacharyya S, Shastri A, Bartenstein M et al (2015) IL8-CXCR2 pathway inhibition as a therapeutic strategy against MDS and AML stem cells. Blood 125:3144-3152

38. Rodríguez-Hernández G, Hauer J, Martín-Lorenzo A, Schäfer D, Bartenhagen C, García-Ramírez I, Auer F, González-Herrero I, Ruiz-Roca L, Gombert M, Okpanyi V, Fischer U, Chen C, Dugas M, Bhatia S, Linka RM, Garcia-Suquia M, Rascón-Trincado MV, Garcia-Sanchez A, Blanco O, García-Cenador MB, García-Criado FJ, Cobaleda C, Alonso-López D, de Las Rivas J, Müschen M, Vicente-Dueñas C, Sánchez-García I, Borkhardt A (2017) Infection exposure promotes ETV6-RUNX1 precursor B-cell leukemia via impaired H3K4 demethylases. Cancer Res 77:43654377

39. Martín-Lorenzo A, Hauer J, Vicente-Dueñas C et al (2015) Infection exposure is a causal factor in B-cell precursor acute lymphoblastic leukemia as a result of Pax5-inherited susceptibility. Cancer Discov 5:1328-1343

40. Skokowa J, Welte K (2009) Dysregulation of myeloid-specific transcription factors in congenital neutropenia: rescue by namptnad+ sirt1. Ann N Y Acad Sci 1176:94-100

41. Friedman AD (2007) Transcriptional control of granulocyte and monocyte development. Oncogene 26:6816-6828

42. Rosenbauer F, Tenen DG (2007) Transcription factors in myeloid development: balancing differentiation with transformation. Nat Rev Immunol 7:105-117

43. Staber PB, Zhang P, Ye M, Welner RS, Levantini E, di Ruscio A, Ebralidze AK, Bach C, Zhang H, Zhang J, Vanura K, Delwel R, Yang H, Huang G, Tenen DG (2014) The Runx-PU.1 pathway preserves normal and AML/ETO9a leukemic stem cells. Blood $124: 2391-2399$

Publisher's note Springer Nature remains neutral with regard to jurisdictional claims in published maps and institutional affiliations. 